\title{
Optimization of diffusion spectrum imaging and $q$-ball imaging on clinical MRI system
}

\author{
Li-Wei Kuo, ${ }^{\mathrm{a}}$ Jyh-Horng Chen, ${ }^{\mathrm{a}}$ Van Jay Wedeen, ${ }^{\mathrm{d}}$ and Wen-Yih Isaac Tseng ${ }^{\mathrm{b}, \mathrm{c}, *}$ \\ anterdisciplinary MRI/MRS Lab, Department of Electrical Engineering, National Taiwan University, Taipei, Taiwan \\ ${ }^{\mathrm{b}}$ Department of Medical Imaging, National Taiwan University Hospital, Taipei, Taiwan \\ ${ }^{\mathrm{c}}$ Center for Optoelectronic Biomedicine, National Taiwan University College of Medicine, Taipei, Taiwan \\ ${ }^{\mathrm{d}}$ Department of Radiology, MGH Martinos Center for Biomedical Imaging, Harvard Medical School, Charlestown, MA, USA
}

Received 16 October 2007; revised 29 January 2008; accepted 14 February 2008

Available online 26 February 2008

\begin{abstract}
Mapping complex crossing fibers using diffusion MRI techniques requires adequate angular precision and accuracy. Beyond diffusion tensor imaging (DTI), high angular resolution sampling schemes such as diffusion spectrum imaging (DSI) and $q$-ball imaging (QBI) were proposed to resolve crossing fibers. These schemes require hundreds of data approximately five to ten times more than DTI, offsetting their clinical feasibility. To facilitate its clinical application, optimum values of highest diffusion sensitivity (bmax) must be investigated under the constraint of scan time and gradient performance. In this study, simulation of human data sets and a following verification experiment were performed to investigate the optimum bmax of DSI and QBI. Four sampling schemes, two with high sampling number, i.e., DSI515 and QBI493, and two with low sampling number, i.e., DSI203 and QBI253, were compared. Deviation angle and angular dispersion were used to evaluate the precision and accuracy among different bmax of each scheme. The results indicated that the optimum bmax was a trade-off between SNR and angular resolution. At their own optimum bmax, the reduced sampling schemes yielded angular precision and accuracy comparable to the high sampling schemes. On our current $3 \mathrm{~T}$ system, the optimum bmax $\left(\mathrm{s} / \mathrm{mm}^{2}\right)$ were 6500 for DSI515, 4000 for DSI203, 3000 for QBI493 and 2500 for QBI253. DSI was incrementally more accurate than QBI, but required a greater demand for gradient performance. In conclusion, our systematic study of optimum bmax in different sampling schemes and the consideration derived wherein could be helpful to determine optimum sampling schemes in other MRI systems.
\end{abstract}

(C) 2008 Elsevier Inc. All rights reserved.

Keywords: Diffusion MRI; Diffusion spectrum imaging; $q$-ball imaging; Optimum parameters

\footnotetext{
* Corresponding author. Center for Optoelectronic Biomedicine, National Taiwan University College of Medicine, No. 1, Sec 1, Jen-Ai Rd., Taipei 100, Taiwan. Fax: +886223926922.

E-mail address: wytseng@ntu.edu.tw (W.-Y.I. Tseng).

Available online on ScienceDirect (www.sciencedirect.com).
}

\section{Introduction}

Diffusion MRI has been widely used to assess the integrity of axonal fibers because of its unique ability to map fiber orientations in vivo (Le Bihan, 2003; Mori and van Zijl, 2002). To measure fiber orientation, diffusion tensor was proposed to estimate the probability distribution of water molecules using 3-dimensional (3-D) Gaussian approximation, from which the principal direction of the tensor was inferred to the fiber orientation (Basser et al., 1994; Pierpaoli et al., 1996). This method, called diffusion tensor imaging (DTI), can accurately define the fiber orientation of a voxel containing fibers with coherent directions, but cannot define directions of heterogeneous fibers presented with crossing or kissing patterns (Frank, 2001, 2002; Tuch et al., 2002; Wiegell et al., 2000). To address this problem, high angular resolution sampling schemes such as diffusion spectrum imaging (DSI) and $q$-ball imaging (QBI) were proposed to resolve local crossing fibers within each voxel (Gilbert et al., 2006a,b; Lin et al., 2003b; Schmahmann et al., 2007; Tuch, 2004; Tuch et al., 2003, 2005; Wedeen et al., 2005). Typically, these methods sample hundreds of data, approximately five to ten times more than DTI, offsetting its advantage in clinical applications (Hagmann et al., 2006; Khachaturian et al., 2007). Recently, diffusion MRI has been considered a potential tool to study abnormal connectivity of neural circuit in patients with neuropsychiatric disease (Ciccarelli et al., 2006; Ge et al., 2005; Jones et al., 2006; Kubicki et al., 2007). In addition, diffusion MRI and especially high $b$-value and angular resolution techniques are important to study normal and abnormal neural circuitry (Hagmann et al., 2007). It is a timely need to investigate the optimum setting of DSI and QBI for clinical scanners.

To perform DSI, we need hundreds of diffusion-attenuated images with variable directions and strengths of diffusion-sensitive gradients (Lin et al., 2003b; Wedeen et al., 2005). A spectral bandwidth (bmax) larger than $10,000 \mathrm{~s} / \mathrm{mm}^{2}$ is recommended to sample diffusionencoding points over the 3-D $q$-space so that the probability density function (PDF) with sufficient resolution and field-of-view (FOV) can 
be obtained (Wedeen et al., 2005). High sampling number of DSI prolongs the scan time, making implementation of this method more susceptible to motion-induced errors (Jiang et al., 2002). Using high bmax poses a stringent challenge to the gradient performance in current clinical systems (Le Bihan et al., 2006). In addition, the high bmax used in clinical scanners resulted in low signal-to-noise ratio (SNR) due to prolonged TE and substantial diffusion-induced signal decay (Meca et al., 2004). Poor SNR affects the accuracy of PDF orientation and consequently the accuracy of fiber orientation. In order to overcome these limitations, one approach is to reduce the number of the diffusion-encoding gradients as well as the bmax of DSI. For example, by reducing the routine number of diffusionencoding gradients from 515 to 203 , the scan time can be reduced from approximately $1 \mathrm{~h}$ to $30 \mathrm{~min}$. By lowering bmax, the maximum diffusion gradient strength can be reduced to secure gradient stability. Moreover, diffusion time and TE can be reduced to provide better SNR for the diffusion-weighted images.

More efficient than DSI, QBI samples data on a shell of a constant $b$-value in the $q$-space (Tuch, 2004; Tuch et al., 2003). Typically, its bmax and number of gradient encoding are approximately two- to three-fold lower than DSI, thus is considered more feasible in clinical applications. In QBI, orientation distribution function (ODF) along each radial direction is derived and the local fiber orientation can be inferred by the local maxima of ODF at each voxel.

Although QBI and DSI with reduced bmax and encoding number are potentially advantageous for reducing scan time and improving gradient stability, insufficient sampling rate and inadequate bmax over the $q$-space may lead to inaccurately estimating fiber orientations. For DSI, insufficient sampling rate within the 3-D $q$-space may result in aliasing in the PDF profile. On the other hand, inadequate bmax may result in truncation in Fourier transform, causing a ringing artifact to PDF (Wedeen et al., 2005). As for QBI, it is known that the resolution of ODF depends on the bmax. Accordingly, reduced bmax may degrade the angular resolution of QBI (Tuch, 2004). All the above problems may lead to inaccurately estimating local fiber orientation. Therefore, a systematic study on how to determine the optimum bmax and encoding number for clinical application is needed.

To facilitate clinical application, it is necessary to investigate optimum values of bmax under the constraint of scan time and gradient performance on current clinical system. Thus, the purpose of this study is to determine the optimum sampling scheme for DSI and QBI obtained from $3 \mathrm{~T}$ clinical system. In either DSI or QBI, one scheme with a higher encoding number (approximately 500) and one with a lower encoding number (approximately 200) were studied. For each scheme, the precision and accuracy of fiber orientation were quantified and compared between different bmax values. Since it is exhausting to perform all the experiments on clinical system, simulation from human data sets was first performed to determine the optimum parameters. Based on the simulation results, selective ranges of optimum bmax for each sampling scheme were decided for the verification study. Finally, the combined effects of gradient number and bmax on the angular resolution of DSI and QBI were discussed and the strategy of determining optimum sampling schemes on clinical scanners was recommended.

\section{Materials and methods}

\section{Diffusion spectrum imaging (DSI) and q-ball imaging (QBI)}

The concept of DSI is based on the Fourier relationship between the attenuated echo signal in $q$-space $E(q)$ and the probability density function (PDF) of water molecular diffusion $P_{\mathrm{s}}(r)$

$E(q)=\int P_{\mathrm{s}}(R, \Delta) \exp (i 2 \pi q R) \mathrm{d} R$,

where $R$ is the relative displacement of water molecular diffusion during the diffusion time (Callaghan, 1991). Based on this relationship, 3-D Fourier transform of the echo signal over the $q$-space yields the 3-D PDF (Wedeen et al., 2005). In practice, the diffusion spectrum is reconstructed by applying the 3-D discrete Fourier transform to the grid $q$-space data $E(q)$. For each voxel, the attenuated echo signals are filled into the 3 -D Cartesian coordinate space $(17 \times 17 \times 17)$ according to their respective position vectors. As suggested by Wedeen et al. (2005), a Hanning window is used to smooth the attenuated echo signal to prevent the truncation artifact. In our analysis, the Hanning window with a raised cosine function, $h(r)=0.5 \times \cos (2 \pi r / 17)$, was applied for all the DSI schemes. After 3-D Fourier transform applied on $E(q)$, a discrete 3-D PDF space can be derived in a 3-D Cartesian coordinate space $(17 \times 17 \times 17)$. In order to characterize the magnitude of directional diffusion probability, orientation distribution function (ODF) was then calculated based on the following definition. The definition of the ODF in the direction of the unit vector $\mathbf{u}$ for DSI is

$\operatorname{ODF}(\mathbf{u})=\int_{0}^{r_{\max }} P_{\mathrm{s}}(r \mathbf{u}) r^{2} \mathrm{~d} r$.

This approach can be viewed as a weighted radial summation of $P_{\mathrm{s}}$ and the local fiber orientations were inferred by the orientations of the local maxima of ODF (Lin et al., 2003b; Wedeen et al., 2005).

QBI is reconstructed based on the relationship of the interested ODF vector and its orthogonal plane projected onto the acquired $q$-space data, so-called Funk-Radon transform (Tuch, 2004; Tuch et al., 2003, 2005). The ODF was directly calculated from the attenuated echo signal on a shell in the $q$-space with a fixed $b$-value based on the Funk-Radon transform approach. The detailed procedures of QBI reconstruction can be found in Tuch's papers, and were described very briefly here. It bypasses the computation of PDF and estimates ODF and local fiber orientations directly. To derive ODF in a desired direction, the circular integral is performed along the equator whose plane is perpendicular to this particular ODF direction based on the following equation:

$\operatorname{ODF}(\mathbf{u})=\frac{1}{Z} \int_{q \perp \mathbf{u}} E(q, \Delta) \mathrm{d} q$,

where $\mathbf{u}$ is the unit vector for the desired ODF direction and $Z$ is the normalization constant. In practice, the signals on the equator have to be interpolated and the interpolation kernel width $(\sigma)$ closely affects the accuracy of the ODF estimation. According to Tuch's simulation results, we performed QBI reconstruction using $\sigma=5^{\circ}$ to achieve a trade-off between the accuracy and stability (Tuch, 2004). To further improve ODF accuracy, appropriate smoothing function was applied to the estimated ODF. To simplify the comparison, a simple average smoothing function with the same smoothing window was performed to post-process the QBI data. For both DSI and QBI, ODF within each voxel was reconstructed to 162 radial directions pointing at the vertices of regular triangular mesh on the unit sphere surface. Reconstruction of DSI and QBI data was performed using an in-house program written in MATLAB 7.0 (The Mathworks, Natick, MA, USA). 
Table 1

The radii and encoding numbers of DSI schemes

\begin{tabular}{lcccc}
\hline & DSI925 & DSI691 & DSI515 & DSI203 \\
\hline Radius $(r)$ & 6.0 & 5.4 & 5.0 & 3.6 \\
Encoding numbers & 925 & 691 & 515 & 203 \\
\hline
\end{tabular}

Simulation

To optimize bmax for different DSI or QBI encoding schemes, simulation from in vivo human data was performed to estimate the optimum range of bmax. The simulation consisted of three procedures: subsampling, reconstruction and angular analysis. A total of five volunteers were recruited in the simulation study. For each volunteer, a DSI data set of high sampling rate and bandwidth in $q$-space was acquired to support the full sampling reference, subsampled DSI and QBI data. In the present study, a DSI data set with 925 encoding grid points over the 3-D $q$-space (DSI925) with bmax of $9000 \mathrm{~s} / \mathrm{mm}^{2}$ was acquired. Considering sufficient SNR, we chose 691 grid points (DSI691) with the same sampling rate as DSI925 in the $q$-space to serve as the full sampling reference. Theoretically, using higher bmax can achieve better angular resolution to resolve fiber crossing and can reduce the truncation artifact in 3-D Fourier transform. In order to achieve high bmax on clinical scanners, however, long $\delta$ and $\Delta$ have to be applied, and this leads to long TE and consequently reduced SNR. Insufficient SNR may bias the estimated orientation and offset the advantages brought by using high bmax values. In fact, we found that fiber vectors reconstructed from DSI925 with bmax of $9000 \mathrm{~s} / \mathrm{mm}^{2}$ showed large noise fluctuation, whereas fiber vectors produced from DSI691 with bmax of $7000 \mathrm{~s} /$ $\mathrm{mm}^{2}$ were reasonable and reproducible. Based on this observation, we decided to use the DSI691 data set as a reference in the simulation study. Therefore, the rationale of choosing DSI691 as the reference is that DSI691 with bmax of $7000 \mathrm{~s} / \mathrm{mm}^{2}$ is the data set attainable on our current system with sufficiently high bmax and sampling density without compromising SNR.

Four plausible DSI and QBI encoding schemes were investigated, and their optimum settings were determined by comparing them with the full sampling reference. For DSI, two fixed gradient numbers, 203 (DSI203) and 515 (DSI515), were subsampled with bmax of 1000, 2000, 3000, 4000, 5000, 6000 and $7000 \mathrm{~s} / \mathrm{mm}^{2}$. For DSI schemes, the position vectors are the entire grid points $\left(q_{\mathrm{x}}, q_{\mathrm{y}}, q_{\mathrm{z}}\right)$ over the 3-D $q$-space under the relationship that $\left(q_{\mathrm{x}}^{2}+q_{\mathrm{y}}^{2}+q_{\mathrm{z}}^{2}\right) \leq r^{2}$, where $r$ is the radius specified for a DSI encoding scheme. The DSI encoding schemes and specified radii are shown in Table 1. To obtain QBI data sets with comparable subsampled numbers, including one null image with no diffusion weighting, 252 (QBI253) and 492 (QBI493) gradient numbers were subsampled with the same bmax values as those for DSI subsampling schemes. For QBI schemes, the position vectors are sampled
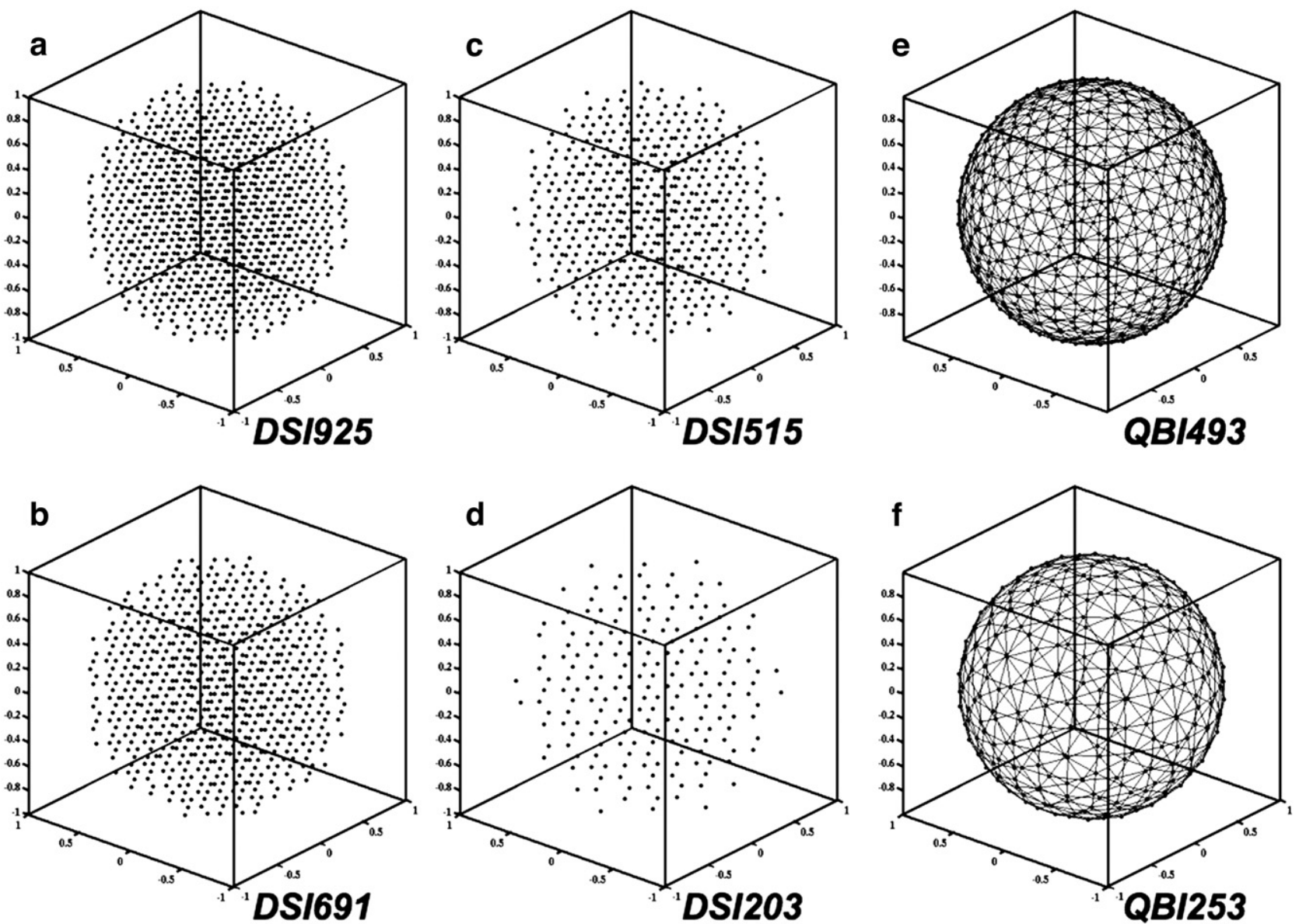

Fig. 1. Illustration of the encoding schemes over the $q$-space. Panels a-f correspond to DSI925, DSI691 (full sampling reference), DSI515, DSI203, QBI493 and QBI253, respectively. The maximum $q$-value was normalized to unit for each plot. In simulation, all of the encoding schemes from b to f were subsampled from DSI925. In verification study, data sets of DSI691 (b) and one of four encoding schemes from c to f were acquired. 
symmetrically on a unit sphere in the $q$-space and tessellated from icosahedrons. Specifically, the encoding points of QBI493 and QIBI253 are obtained from the vertices of seven-fold and five-fold tessellated icosahedrons, respectively. All of the subsampled data sets were obtained by performing linear interpolation on the DSI925 data set using MATLAB built-in function. The full sampling reference, DSI691, was a subset of DSI925 without performing interpolation. Note that for each encoding scheme, the number of encoding points was fixed, and bmax was the only variable. Fig. 1 illustrates the encoding schemes of DSI925, full sampling reference (DSI691) and four subsampled DSI and QBI, showing, respectively, grid and spherical encoding points over the $q$-space. After subsampling, all subsampled data sets and full sampling reference were reconstructed to determine the local fiber orientations.

The angular precision and accuracy of fiber orientation were assessed by the deviation angle and angular dispersion between the reference and the four schemes. Voxels in the cerebral white matter were selected for comparison, the white matter was segmented by setting the threshold of the diffusion anisotropy (DA) of the full sampling reference (Kuo et al., 2003). These voxels were categorized into two groups, one containing single fiber and one containing crossing fibers. The number of local maxima orientations derived from the full sampling reference was used to categorize the single-fiber or crossing-fiber groups. For the angular analysis, only the first two longest local maxima vectors were compared. This led to four types of combinations; as illustrated in Fig. 2a, types 1 and 2 for single-fiber group and types 3 and 4 for crossing-fiber group. For types 1 and 2, the pair of $\mathbf{v}_{\mathrm{r} 1}$ and $\mathbf{v}_{\mathrm{c} 1}$ was used to evaluate the angular precision and dispersion. For type 3, both $\mathbf{v}_{\mathrm{r} 1}$ and $\mathbf{v}_{\mathrm{r} 2}$ were compared with $\mathbf{v}_{\mathrm{c} 1}$, resulting in two pairs for analysis; for type 4, two pairs were used for analysis, $\left(\mathbf{v}_{\mathrm{r} 1}, \mathbf{v}_{\mathrm{c} 1}\right)$ and $\left(\mathbf{v}_{\mathrm{r} 2}, \mathbf{v}_{\mathrm{c} 2}\right)$. Note that in the crossingfiber group only the voxels with the ODF ratio higher than 0.7 were used for angular analysis, the ODF ratio was defined as the ratio of length of the second longest vector to that of the first longest vector.

To perform angular analysis for single- and crossing-fiber groups, vector transformation was applied to each pair; a rotating matrix which rotated the reference vector of each pair to align with the $z$-axis was determined and the same matrix was applied to the compared vector (Fig. 2b). After this step, all the compared vectors were distributed around the $z$-axis (Fig. 2c). The distribution on the unit hemisphere of these transformed compared vectors was assumed unimodal (Mardia and Jupp, 2000).

After vector transformation, two indices were derived to evaluate the angular precision and angular dispersion for both single-fiber and crossing-fiber groups. Angular precision $\left(P_{\mathrm{a}}\right)$ was defined as the mean of the angles distended by the individual transformed compared vectors and the reference vector,

$P_{\mathrm{a}}=\frac{1}{n} \sum_{i=1}^{n} \theta_{i}$,

where $\theta_{i}$ was the deviation angle between $i$ th transformed vector and the reference vector and $n$ was the total number of transformed vectors. Angular dispersion $(D)$ was defined as the first eigenvalue of the scatter matrix $(\mathbf{S})$, where

$S=\frac{1}{n} \sum_{i=1}^{n} v_{i} v_{i}^{T}$,

$\mathbf{v}_{i}$ was the $i$ th transformed vector, $T$ represented the vector transpose function, and $n$ was the total number of transformed vectors (Mardia and Jupp, 2000). This index indicated the degree of dispersion of these vectors on a unit sphere, and was used to investigate the uncertainty of mapping fiber orientations or angular accuracy. In fact, the quantity $P_{\mathrm{a}}$ in our study is the mean deviation angle, and is
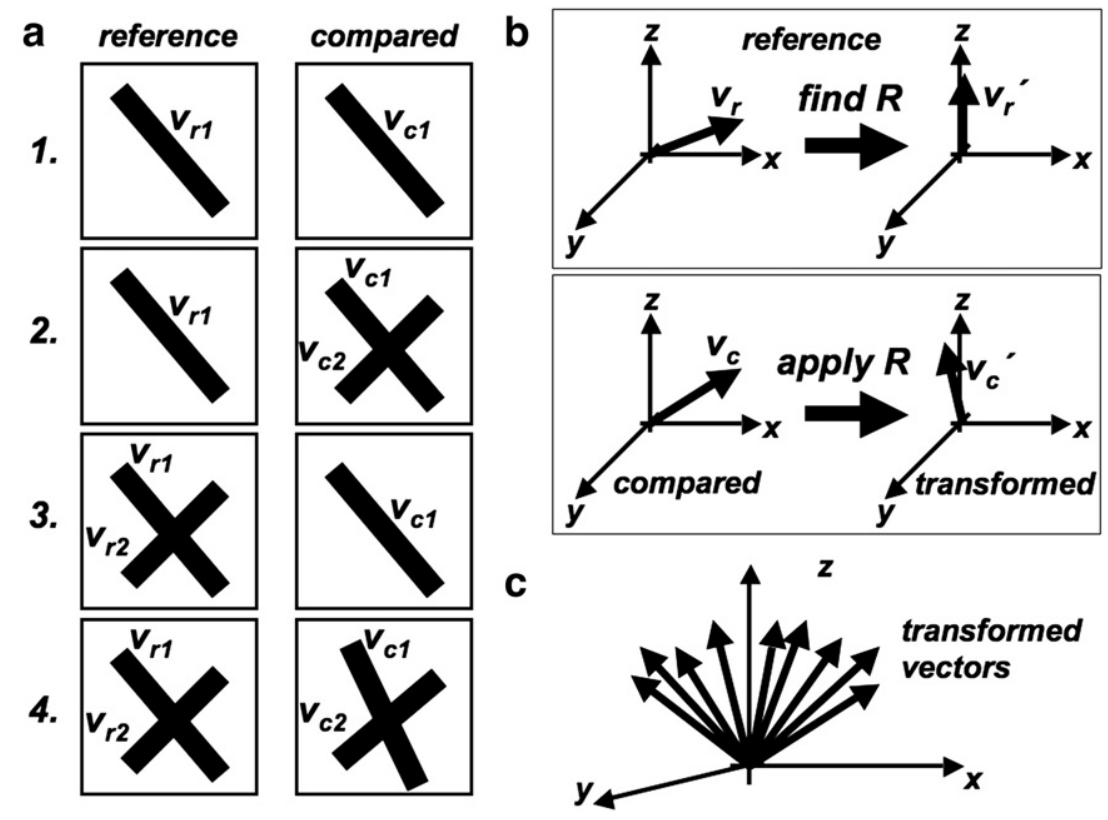

Fig. 2. Procedures of angular analysis. (a) Four types of combination for single-fiber (1-2) and crossing-fiber (3-4) groups. The left column represents the fibers in a voxel of the full sampling reference and the right column represents the fibers in the same voxel position of the compared DSI or QBI data set. The vectors in the full sampling reference and in the compared data were denoted, respectively, by the subscripts $\mathrm{r}$ and $\mathrm{c}$, and the first and second longest local maxima vectors were denoted respectively by the subscripts 1 and 2. (b) Transformation of the reference vectors and compared vectors. The rotating matrix $\mathbf{R}$ was determined by the transformation from $\mathbf{v}_{\mathrm{r}}$ to $\mathbf{v}_{\mathrm{r}}^{\prime}$, $\mathbf{R}$ was then applied to the corresponding compared vector $\mathbf{v}_{\mathrm{c}}$ to obtain $\mathbf{v}_{\mathrm{c}}^{\prime}$. (c) Distribution of the transformed compared vectors on the unit hemisphere. 
Table 2

Sequence parameters for DSI and QBI encoding schemes

\begin{tabular}{|c|c|c|c|c|c|c|}
\hline Schemes & Field-of-view (mm) & Matrix size $\times$ slice numbers & Voxel dimensions (mm) & $\mathrm{TR}$ (ms) & $\Delta / \delta(\mathrm{ms})$ & $g_{\max }(\mathrm{mT} / \mathrm{m})$ \\
\hline DSI925 (simulation) & $350 \times 350$ & $128 \times 128 \times 15$ & $2.7 \times 2.7 \times 2.7$ & 2900 & $85 / 38$ & 35 \\
\hline $\begin{array}{l}\text { DSI691/DSI515/DSI203/ } \\
\text { QBI493/QBI253 (verification) }\end{array}$ & $350 \times 350$ & $128 \times 128 \times 9$ & $2.7 \times 2.7 \times 2.7$ & 2000 & $80 / 35$ & 35 \\
\hline
\end{tabular}

Healthy volunteers were studied in the simulation $(N=5)$ and verification studies $(N=8)$.

equivalent to the angular resolution in terms of deviation from this direction in degree for a given reference direction. The quantity $D$ is a metric indicating the orientation spread function, implying the capability of resolving two directions of diffusion maximum.

To summarize our simulation approach, first, in vivo human data of high sampling density and bmax (DSI925) were acquired. A full sampling reference (DSI691) with bmax of $7000 \mathrm{~s} / \mathrm{mm}^{2}$ and four subsampling schemes (DSI515, DSI203, QBI493 and QBI253) were obtained by interpolating DSI925. Different bmax from 1000 to $7000 \mathrm{~s} / \mathrm{mm}^{2}$ with a step size of $1000 \mathrm{~s} / \mathrm{mm}^{2}$ was investigated to determine the optimum bmax for each subsampling scheme. To evaluate the angular precision and accuracy between each scheme and the full sampling reference, two indices, $P_{\mathrm{a}}$ and $D$, were derived for both single- and crossing-fiber groups. In simulation, the results among five subjects were averaged to determine the optimum range of bmax for each scheme, according to which a verification study was performed.

\section{Verification study}

To confirm the simulation results, a verification study was performed on other eight normal volunteers. In addition to the DSI and QBI schemes used in the simulation, another comparison was performed among schemes using the minimum echo time (TE) to attribute the best SNR for each scheme. Therefore, a total of eight schemes were compared, including DSI203, DSI515, QBI253 and QBI493 with the same TE as the full sampling reference, and those with the minimum TE. The range of bmax for each sampling scheme was decided based on the simulation results, which reduced the total scan time for each subject to prevent artifacts due to long scan time, such as motion or $B_{0}$ shift. Similar to the simulation, angular precision and accuracy were analyzed, from which the optimum bmax for each sampling scheme was determined.

\section{$M R$ experiments}

MR experiments were performed on a $3 \mathrm{~T}$ MRI system with the maximum gradient strength of $40 \mathrm{mT} / \mathrm{m}$ and maximum slew rate along a single direction of $200 \mathrm{mT} / \mathrm{m} / \mathrm{ms}$ (Trio, Siemens, Erlangen, Germany). To secure gradient power supply, the maximum diffusion gradient strength was restricted to $35 \mathrm{mT} / \mathrm{m}$. A Siemens eightchannel head array coil was used as a receiver and no parallel imaging technique was applied in this study. Subjects' heads were fixed by a vacuum sponge. To reduce the eddy current effect, twicerefocused balanced spin-echo diffusion echo-planer imaging (EPI) sequence was used to acquire MR diffusion-weighted images (DWI) (Reese et al., 2003). The protocol was approved by the hospital ethics committee and informed consent was obtained from all the volunteers. In the simulation study, MR experiments were performed on five healthy adult volunteers (four males and one female, 24-35 years old). Note that only one DSI data set (DSI925) with bmax of $9000 \mathrm{~s} / \mathrm{mm}^{2}$ was acquired in the simulation study for each volunteer. Fifteen slices were acquired covering the middle cerebrum with TR of $2900 \mathrm{~ms}$, resulting in the total scan time of approximately $60 \mathrm{~min}$. DWI at the in-plane resolution and slice thickness of $2.7 \mathrm{~mm}$ was obtained with TE minimized to $155 \mathrm{~ms}$, diffusion time $(\Delta)$ of $85 \mathrm{~ms}$ and diffusion gradient duration $(\delta)$ of $38 \mathrm{~ms}$, resulting in that $q_{\max }=\gamma \mathbf{g} \delta=0.57 \mu \mathrm{m}^{-1}$ and $r_{\max }=\Delta q^{-1}=$ $11 \mu \mathrm{m}$, where $\gamma$ is the gyromagnetic ratio and $\mathbf{g}$ is the diffusion gradient vector. In the verification study, MR experiments were performed in eight healthy adult volunteers (two males and six females, 22-35 years old). For each volunteer, we obtained a full sampling reference data set DSI691 with a bmax of $7000 \mathrm{~s} / \mathrm{mm}^{2}$ and a TE/ $\Delta / \delta$ of $147 / 80 / 35 \mathrm{~ms}$ as well as one of the four subsampling schemes with variable values of bmax decided from the simulation study. Note that four volunteers were performed with the same TE as the full sampling reference and the other four were performed with the minimum TE. A total of nine slices covering the middle cerebrum were acquired with a TR of $2000 \mathrm{~ms}$. The minimum TE values were $101,114,123,130,137,142$ and $147 \mathrm{~ms}$, respectively, corresponding to bmax values from 1000 to $7000 \mathrm{~s} / \mathrm{mm}^{2}$ with an equal step size of $1000 \mathrm{~s} / \mathrm{mm}^{2}$. The sequence parameters and encoding schemes are summarized in Tables 2 and 3, respectively.

\section{Results}

\section{Simulation}

Table 4 lists the summary of the simulation study. For the singlefiber group of DSI515, there was a significant difference $(p<0.05)$ of $P_{\mathrm{a}}$ and $D$ between bmax of $6000 \mathrm{~s} / \mathrm{mm}^{2}$ and any other bmax except $7000 \mathrm{~s} / \mathrm{mm}^{2}$. For the crossing-fiber group of DSI515, there was a significant difference $(p<0.05)$ between bmax of $6000 \mathrm{~s} / \mathrm{mm}^{2}$ and any other bmax. For both groups of DSI203, significant differences

Table 3

Summary of TE and corresponding bmax for DSI and QBI encoding schemes

\begin{tabular}{ll}
\hline Schemes & TE(bmax) $\left(\right.$ TE: $\left.\mathrm{ms}, \mathrm{bmax}: \mathrm{s} / \mathrm{mm}^{2}\right)$ \\
\hline DSI925 & $155(9000)$ \\
DSI691 & $147(7000)$ \\
DSI515, sameTE & $147(5000,6000,7000)$ \\
DSI515, minTE & $137(5000), 142(6000), 147(7000)$ \\
DSI203, sameTE & $147(1000,2000,3000,4000,5000)$ \\
DSI203, minTE & $101(1000), 114(2000), 123(3000)$, \\
& $130(4000), 137(5000), 142(6000)$ \\
QBI493, sameTE & $147(2000,3000,4000,5000)$ \\
QBI493, minTE & $114(2000), 123(3000), 130(4000), 137(5000)$ \\
QBI253, sameTE & $147(1000,2000,3000,4000,5000)$ \\
QBI253, minTE & $101(1000), 114(2000), 123(3000)$, \\
& $130(4000), 137(5000), 142(6000)$ \\
\hline
\end{tabular}

"sameTE" means a series of study performed with the same TE for all bmax, "minTE" means a series of study performed with the minimum TE for individual bmax. 
Table 4

Summary of simulation results

\begin{tabular}{|c|c|c|c|c|c|c|c|c|c|}
\hline \multirow[t]{2}{*}{ Scheme } & & & \multicolumn{7}{|c|}{ bmax $(\mathrm{s} / \mathrm{mm} 2)$} \\
\hline & & & 1000 & 2000 & 3000 & 4000 & 5000 & 6000 & 7000 \\
\hline \multirow[t]{4}{*}{ DSI515 } & Single-fiber & $P_{\mathrm{a}}\left({ }^{\circ}\right)$ & $53.72 \pm 3.92$ & $21.46 \pm 2.31$ & $10.11 \pm 0.83$ & $6.98 \pm 0.32$ & $4.53 \pm 0.15$ & $2.64 \pm 0.09$ & $2.67 \pm 0.14$ \\
\hline & & $D$ & $0.402 \pm 0.033$ & $0.816 \pm 0.030$ & $0.936 \pm 0.007$ & $0.960 \pm 0.002$ & $0.976 \pm 0.001$ & $0.986 \pm 0.001$ & $0.986 \pm 0.001$ \\
\hline & Crossing-fiber & $P_{\mathrm{a}}\left({ }^{\circ}\right)$ & $57.88 \pm 0.40$ & $44.33 \pm 1.41$ & $33.73 \pm 0.94$ & $27.17 \pm 1.17$ & $21.69 \pm 1.12$ & $16.53 \pm 0.93$ & $18.07 \pm 1.29$ \\
\hline & & $D$ & $0.354 \pm 0.005$ & $0.513 \pm 0.020$ & $0.644 \pm 0.010$ & $0.719 \pm 0.013$ & $0.777 \pm 0.013$ & $0.829 \pm 0.011$ & $0.811 \pm 0.015$ \\
\hline \multirow[t]{4}{*}{ DSI203 } & Single-fiber & $P_{\mathrm{a}}\left({ }^{\circ}\right)$ & $17.15 \pm 1.08$ & $9.83 \pm 0.23$ & $4.25 \pm 0.19$ & $3.91 \pm 0.35$ & $5.30 \pm 0.78$ & $6.43 \pm 1.24$ & $7.71 \pm 1.63$ \\
\hline & & $D$ & $0.870 \pm 0.013$ & $0.940 \pm 0.002$ & $0.977 \pm 0.001$ & $0.979 \pm 0.002$ & $0.970 \pm 0.006$ & $0.962 \pm 0.010$ & $0.952 \pm 0.014$ \\
\hline & Crossing-fiber & $P_{\mathrm{a}}\left({ }^{\circ}\right)$ & $40.99 \pm 0.88$ & $31.67 \pm 0.89$ & $22.09 \pm 1.00$ & $23.09 \pm 1.81$ & $26.99 \pm 3.37$ & $29.69 \pm 3.59$ & $31.79 \pm 3.61$ \\
\hline & & $D$ & $0.558 \pm 0.012$ & $0.672 \pm 0.011$ & $0.772 \pm 0.013$ & $0.758 \pm 0.021$ & $0.715 \pm 0.040$ & $0.684 \pm 0.042$ & $0.661 \pm 0.042$ \\
\hline \multirow[t]{4}{*}{ QBI493 } & Single-fiber & $P_{\mathrm{a}}\left({ }^{\circ}\right)$ & $5.77 \pm 0.80$ & $6.00 \pm 1.63$ & $10.02 \pm 4.21$ & $12.05 \pm 3.51$ & $13.35 \pm 1.58$ & $15.37 \pm 1.89$ & $18.39 \pm 2.57$ \\
\hline & & $D$ & $0.968 \pm 0.005$ & $0.962 \pm 0.014$ & $0.927 \pm 0.043$ & $0.909 \pm 0.037$ & $0.870 \pm 0.016$ & $0.875 \pm 0.020$ & $0.841 \pm 0.029$ \\
\hline & Crossing-fiber & $P_{\mathrm{a}}\left({ }^{\circ}\right)$ & $28.66 \pm 0.97$ & $27.42 \pm 4.31$ & $32.15 \pm 5.43$ & $34.90 \pm 4.81$ & $36.85 \pm 3.23$ & $38.06 \pm 2.29$ & $40.03 \pm 2.46$ \\
\hline & & $D$ & $0.697 \pm 0.010$ & $0.705 \pm 0.051$ & $0.654 \pm 0.065$ & $0.624 \pm 0.060$ & $0.601 \pm 0.040$ & $0.589 \pm 0.028$ & $0.564 \pm 0.031$ \\
\hline \multirow[t]{4}{*}{ QBI253 } & Single-fiber & $P_{\mathrm{a}}\left({ }^{\circ}\right)$ & $5.87 \pm 0.82$ & $6.16 \pm 1.62$ & $10.06 \pm 4.15$ & $12.15 \pm 3.49$ & $13.54 \pm 1.59$ & $15.51 \pm 1.84$ & $18.74 \pm 2.62$ \\
\hline & & $D$ & $0.967 \pm 0.005$ & $0.961 \pm 0.014$ & $0.926 \pm 0.042$ & $0.908 \pm 0.037$ & $0.895 \pm 0.016$ & $0.874 \pm 0.019$ & $0.836 \pm 0.029$ \\
\hline & Crossing-fiber & $P_{\mathrm{a}}\left({ }^{\circ}\right)$ & $28.61 \pm 1.14$ & $27.31 \pm 4.17$ & $32.42 \pm 5.32$ & $34.81 \pm 4.63$ & $36.52 \pm 3.40$ & $37.99 \pm 2.40$ & $40.37 \pm 2.26$ \\
\hline & & $D$ & $0.699 \pm 0.013$ & $0.708 \pm 0.049$ & $0.651 \pm 0.065$ & $0.625 \pm 0.057$ & $0.606 \pm 0.042$ & $0.590 \pm 0.029$ & $0.560 \pm 0.029$ \\
\hline
\end{tabular}

$(p<0.05)$ were found between bmax of $4000 \mathrm{~s} / \mathrm{mm}^{2}$ and any other bmax except $3000 \mathrm{~s} / \mathrm{mm}^{2}$. For both QBI493 and QBI253, significant differences $(p<0.05)$ were found between bmax of $1000 \mathrm{~s} / \mathrm{mm}^{2}$ and any other bmax except $2000 \mathrm{~s} / \mathrm{mm}^{2}$ in either single-fiber or crossingfiber group.

Fig. 3a shows average values of $P_{\mathrm{a}}$ against different bmax in DSI515. The optimum bmax was found in the range from 6000 to $7000 \mathrm{~s} / \mathrm{mm}^{2}$ in the single-fiber group and $6000 \mathrm{~s} / \mathrm{mm}^{2}$ in the crossingfiber group. As shown in Table 4, the minimum values of $P_{\mathrm{a}}$ averaged over the five subjects was $2.64^{\circ} \pm 0.09^{\circ}$ in the single-fiber group, and $16.53^{\circ} \pm 0.93^{\circ}$ in the crossing-fiber group. Fig. $3 \mathrm{~b}$ shows average values of $D$ against different bmax in DSI515. Consistent with $P_{\mathrm{a}}$, the optimum bmax was found in the range from 6000 to $7000 \mathrm{~s} / \mathrm{mm}^{2}$ in the single-fiber group and $6000 \mathrm{~s} / \mathrm{mm}^{2}$ in the crossing-fiber group. The maximum values of $D$ were $0.986 \pm 0.001$ in the single-fiber group and $0.829 \pm 0.011$ in the crossing-fiber group (Table 4).

Fig. 3c shows average values of $P_{\mathrm{a}}$ against different bmax in DSI203. The optimum bmax was found in the range from 3000 to $4000 \mathrm{~s} / \mathrm{mm}^{2}$ for both single- and crossing-fiber groups. As shown in Table 4, the minimum values of $P_{\mathrm{a}}$ averaged over the five subjects were $3.91^{\circ} \pm 0.35^{\circ}$ in the single-fiber group and $22.09^{\circ} \pm 1.00^{\circ}$ in the crossing-fiber group. Fig. $3 \mathrm{~d}$ shows average values of $D$ against a

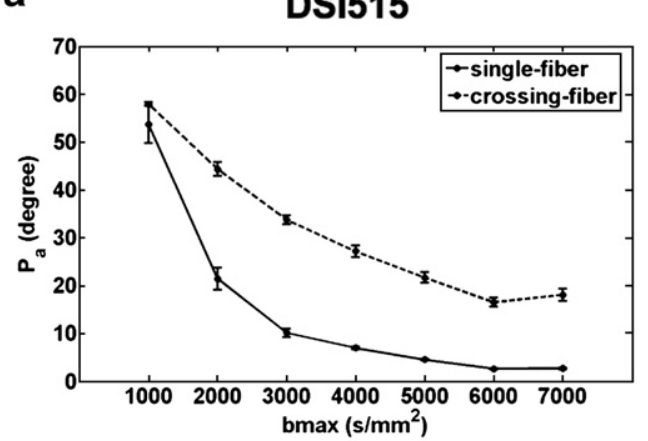

C

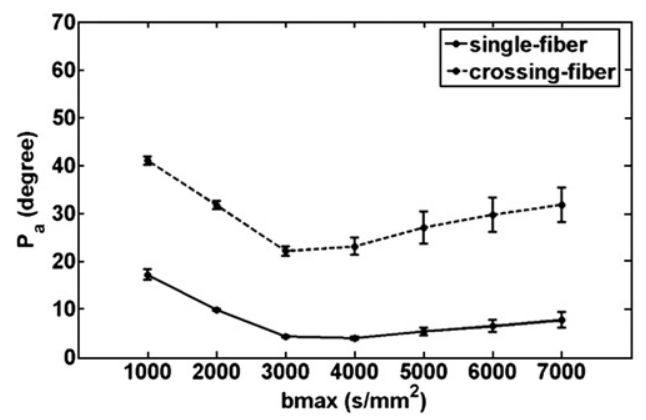

b

DSI515

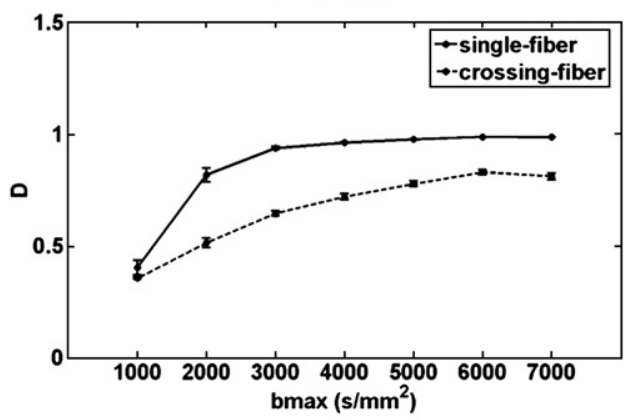

d

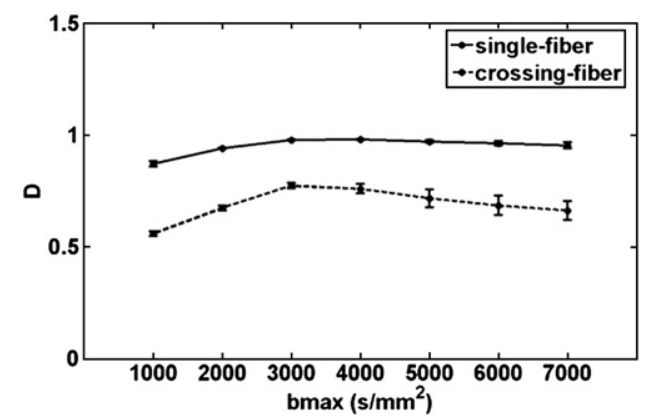

Fig. 3. Angular precision $\left(P_{\mathrm{a}}\right)$ and accuracy $(D)$ against different bmax of the DSI schemes in the simulation study, (a-b) DSI515 and (c-d) DSI203. A total of five subjects were studied. The error bars represent the standard deviation values of $P_{\mathrm{a}}$ and $D$ among these five subjects. 
different bmax in DSI203. Consistent with $P_{\text {a }}$, the optimum bmax was found in the range from 3000 to $4000 \mathrm{~s} / \mathrm{mm}^{2}$ for both single- and crossing-fiber groups. The maximum values of $D$ were $0.979 \pm 0.002$ in the single-fiber group and $0.772 \pm 0.013$ in the crossing-fiber group (Table 4).

Fig. 4 shows the plots of $P_{\mathrm{a}}$ and $D$ against different bmax for both single- and crossing-fiber groups in two QBI schemes, namely, QBI493 and QBI253. Similar tendency of $P_{\mathrm{a}}$ and $D$ was observed in these two schemes; the optimum bmax was found in the range from 1000 to $2000 \mathrm{~s} / \mathrm{mm}^{2}$ for both single- and crossing-fiber groups. In the single-fiber group, the minimum values of $P_{\mathrm{a}}$ and maximum value of $D$ were $5.77^{\circ} \pm 0.80^{\circ}$ and $0.968 \pm 0.005$ for QBI493, $5.87^{\circ} \pm 0.82^{\circ}$ and $0.967 \pm 0.005$ for QBI253. In the crossing-fiber group, they were $27.42^{\circ} \pm 4.31^{\circ}$ and $0.705 \pm 0.051$ for QBI493 and $27.31^{\circ} \pm 4.17^{\circ}$ and $0.708 \pm 0.049$ for QBI352 (Table 4).

\section{Verification study}

Table 5 lists the minimum $P_{\text {a }}$, maximum $D$ and the corresponding bmax for different encoding schemes. For DSI515 (Fig. 5), the optimum bmax values were $7000 \mathrm{~s} / \mathrm{mm}^{2}$ under same TE and $6000 \mathrm{~s} /$ $\mathrm{mm}^{2}$ under minimum TE in the single-fiber group, $7000 \mathrm{~s} / \mathrm{mm}^{2}$ under same TE and $5000 \mathrm{~s} / \mathrm{mm}^{2}$ under minimum TE in the crossingfiber group. For DSI203 (Fig. 6), the optimum bmax values were $4000 \mathrm{~s} / \mathrm{mm}^{2}$ for both same TE and minimum TE cases, and in both single- and crossing-fiber group.

For QBI493 (Fig. 7), the optimum bmax values were $2000 \mathrm{~s} / \mathrm{mm}^{2}$ under both same TE and minimum TE in the single-fiber group; they were $3000 \mathrm{~s} / \mathrm{mm}^{2}$ in the crossing-fiber group. For QBI253 (Fig. 8), they were $1000 \mathrm{~s} / \mathrm{mm}^{2}$ under same TE and $2000 \mathrm{~s} / \mathrm{mm}^{2}$ under minimum TE in the single-fiber group, and they were $2000 \mathrm{~s} / \mathrm{mm}^{2}$ under same TE and $3000 \mathrm{~s} / \mathrm{mm}^{2}$ under minimum TE in the crossing-fiber group.
Table 5

Summary of the verification results

\begin{tabular}{|c|c|c|c|c|c|c|}
\hline \multirow[t]{2}{*}{ Scheme } & \multicolumn{3}{|c|}{ Single-fiber } & \multicolumn{3}{|c|}{ Crossing-fiber } \\
\hline & $P_{\mathrm{a}}\left({ }^{\circ}\right)$ & $D$ & $\begin{array}{l}\text { bmax } \\
\left(\mathrm{s} / \mathrm{mm}^{2}\right)\end{array}$ & $P_{\mathrm{a}}\left({ }^{\circ}\right)$ & $D$ & $\begin{array}{l}\text { bmax } \\
\left(\mathrm{s} / \mathrm{mm}^{2}\right)\end{array}$ \\
\hline DSI515 (sameTE) & 8.89 & 0.942 & 7000 & 35.33 & 0.619 & 7000 \\
\hline DSI515 ( & 8.11 & 0.948 & 6000 & 1.24 & 0.631 & 5000 \\
\hline DSI203 (sameTE) & 8.53 & 0.945 & 4000 & 35.22 & 0.616 & 4000 \\
\hline DSI203 (minTE) & 10.52 & 0.927 & 4000 & 36.19 & 0.607 & 4000 \\
\hline QBI493 (s & 11.53 & 0.917 & 2000 & 36.47 & 0.606 & 3000 \\
\hline QBI493 (minTE) & 15.06 & 0.881 & 2000 & 41.20 & 0.551 & 3000 \\
\hline QBI253 (sameTE) & 10.43 & 0.932 & 1000 & 35.98 & 0.612 & 2000 \\
\hline QBI253 (minTE) & 11.76 & 0.914 & 2000 & 35.55 & 0.616 & 3000 \\
\hline
\end{tabular}

Only the optimum bmax and its corresponding $P_{\mathrm{a}}$ and $D$ values were shown in this table.

The cases of same TE and minimum TE were denoted by sameTE and minTE, respectively.

Fig. 9 shows ODF maps reconstructed from the full sampling reference and the corresponding optimum sampling schemes in the verification study. For the single-fiber group, all the optimum sampling schemes could map homogeneous white matter structure similar to the full sampling reference, especially in the region of corpus callosum. For the crossing-fiber group, especially in the intersection of corpus callosum and internal capsule, both DSI and QBI optimum sampling schemes achieved similar results with the full sampling reference visually.

\section{Discussion}

In this paper, we employed a systematic approach to determine the optimum bmax for different DSI or QBI sampling schemes on a a

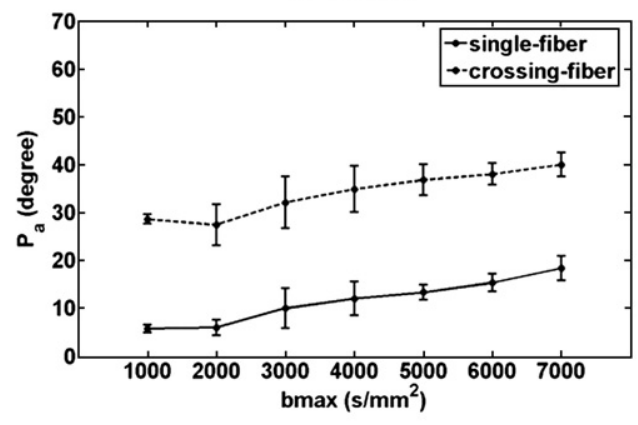

C

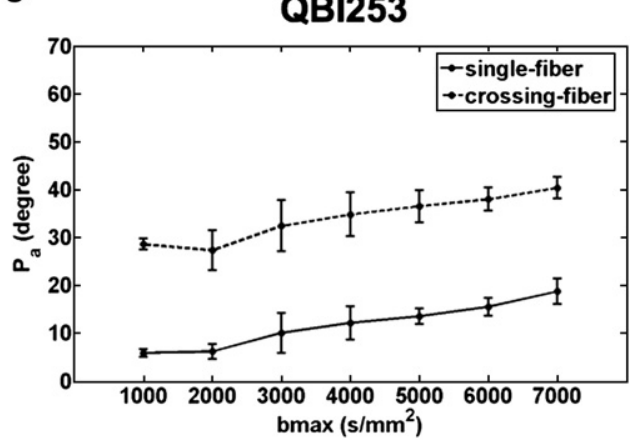

b

QB1493

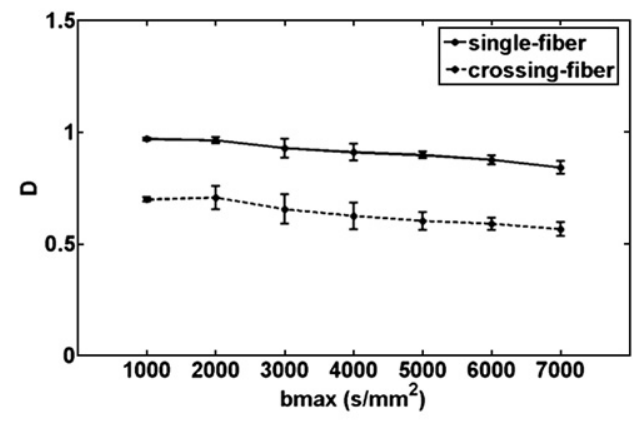

d

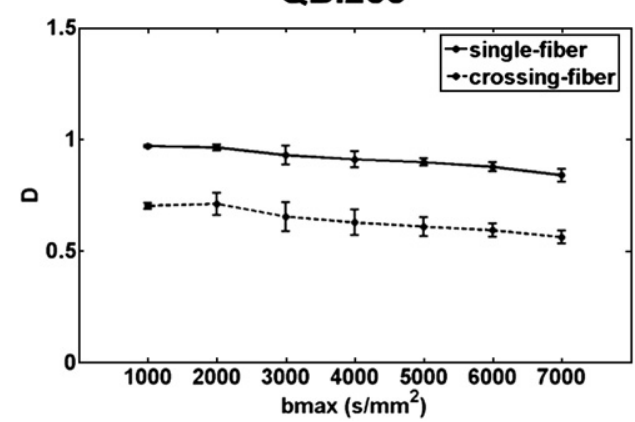

Fig. 4. Angular precision $\left(P_{\mathrm{a}}\right)$ and accuracy $(D)$ against different bmax of the QBI schemes in the simulation study, (a-b) QBI493 and (c-d) QBI253. A total of five subjects were studied. The error bars represent the standard deviation values of $P_{\mathrm{a}}$ and $D$ among these five subjects. 

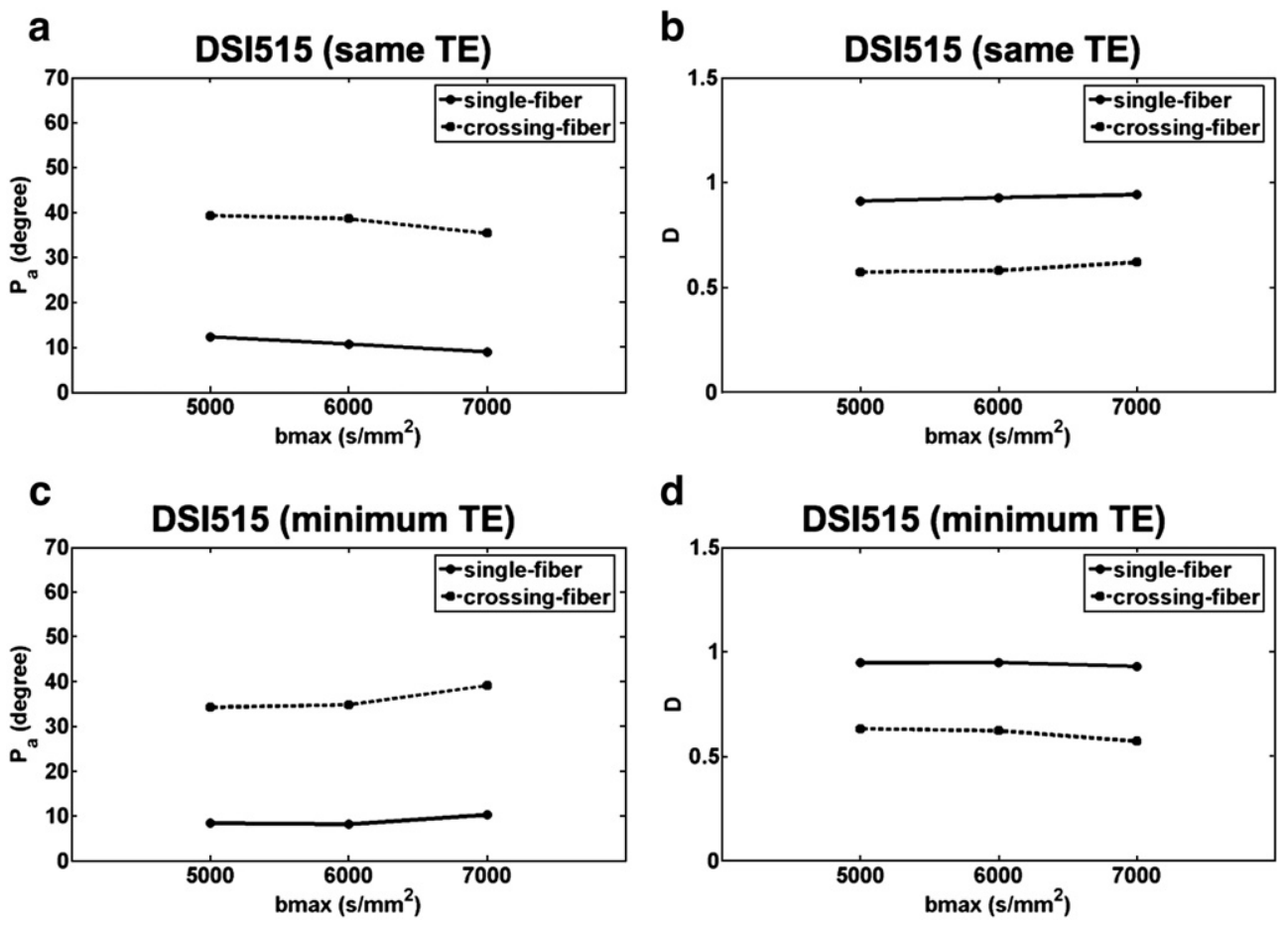

Fig. 5. Angular precision $\left(P_{\mathrm{a}}\right)$ and accuracy $(D)$ against different bmax of DSI515 in the verification study, under same TE (a-b) and minimum TE (c-d). For both cases, a single subject was investigated and the bmax values in the verification study were 5000,6000 and $7000 \mathrm{~s} / \mathrm{mm}^{2}$.

3 T MRI system. We first used human data sets to generate multiple subsampled data sets. The subsampled data sets were categorized according to different sampling numbers and bmax values. The results in the simulation were then verified with in vivo experiments. Under the optimum condition for each sampling scheme, we found that DSI achieved incrementally better angular precision and

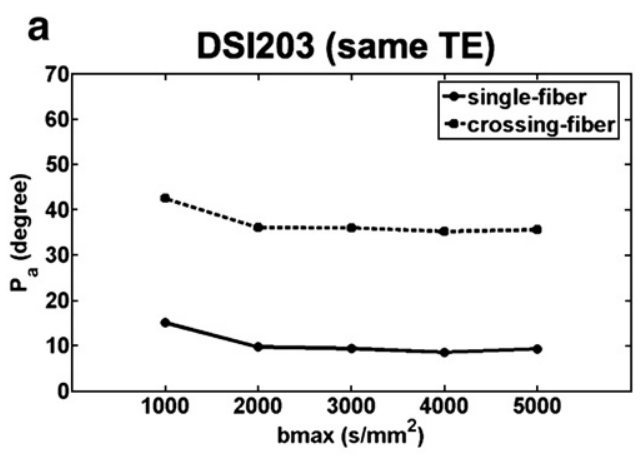

b DSI203 (same TE)
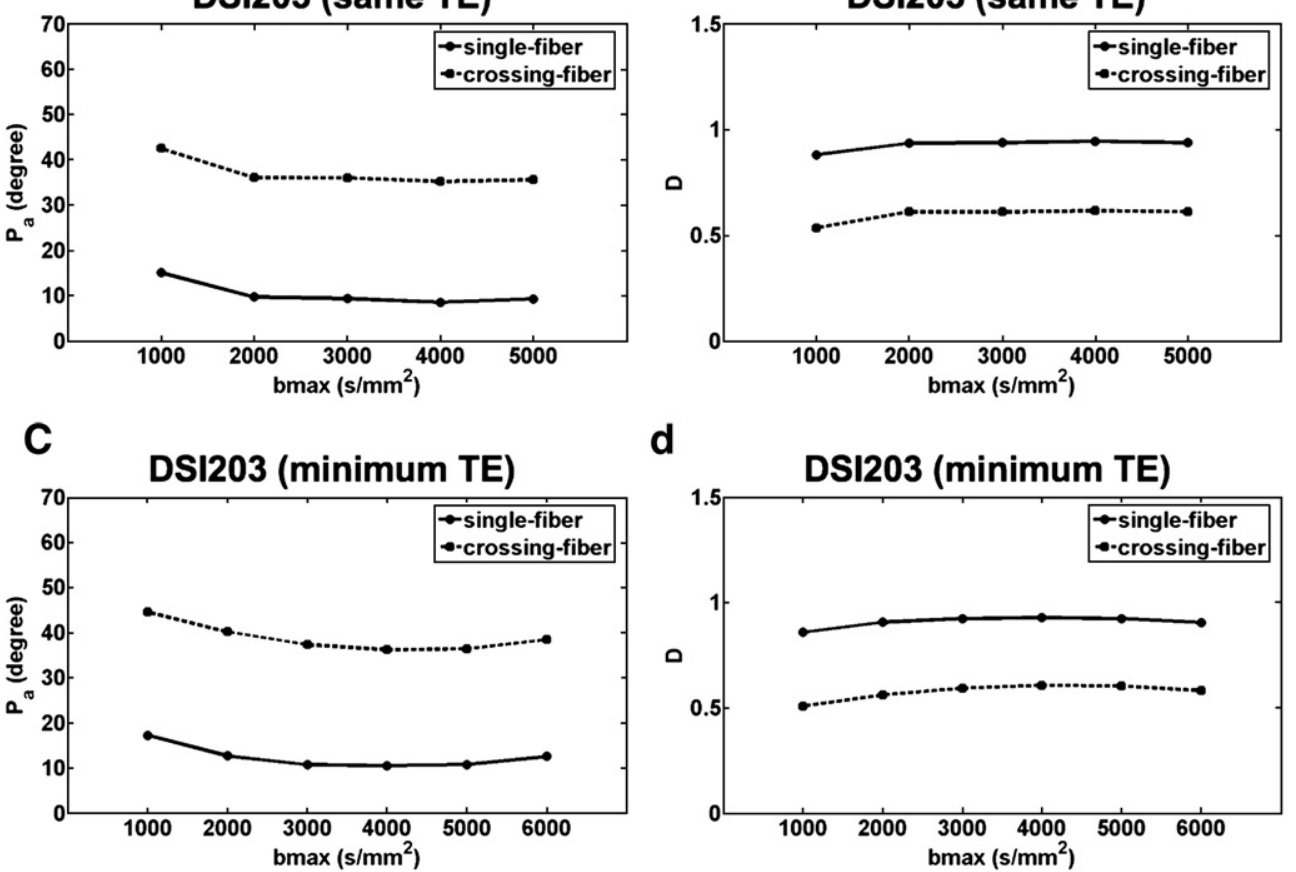

d

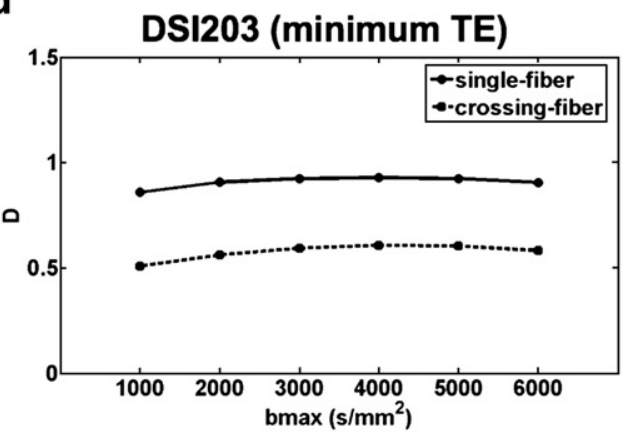

Fig. 6. Angular precision $\left(P_{\mathrm{a}}\right)$ and accuracy $(D)$ against different bmax of DSI203 in the verification study, under same TE (a-b) and minimum TE (c-d). For both cases, a single subject was investigated. The bmax values used in verification study were 1000 to $5000 \mathrm{~s} / \mathrm{mm}^{2}$ for same TE and 1000 to $6000 \mathrm{~s} / \mathrm{mm}^{2}$ for minimum TE. 

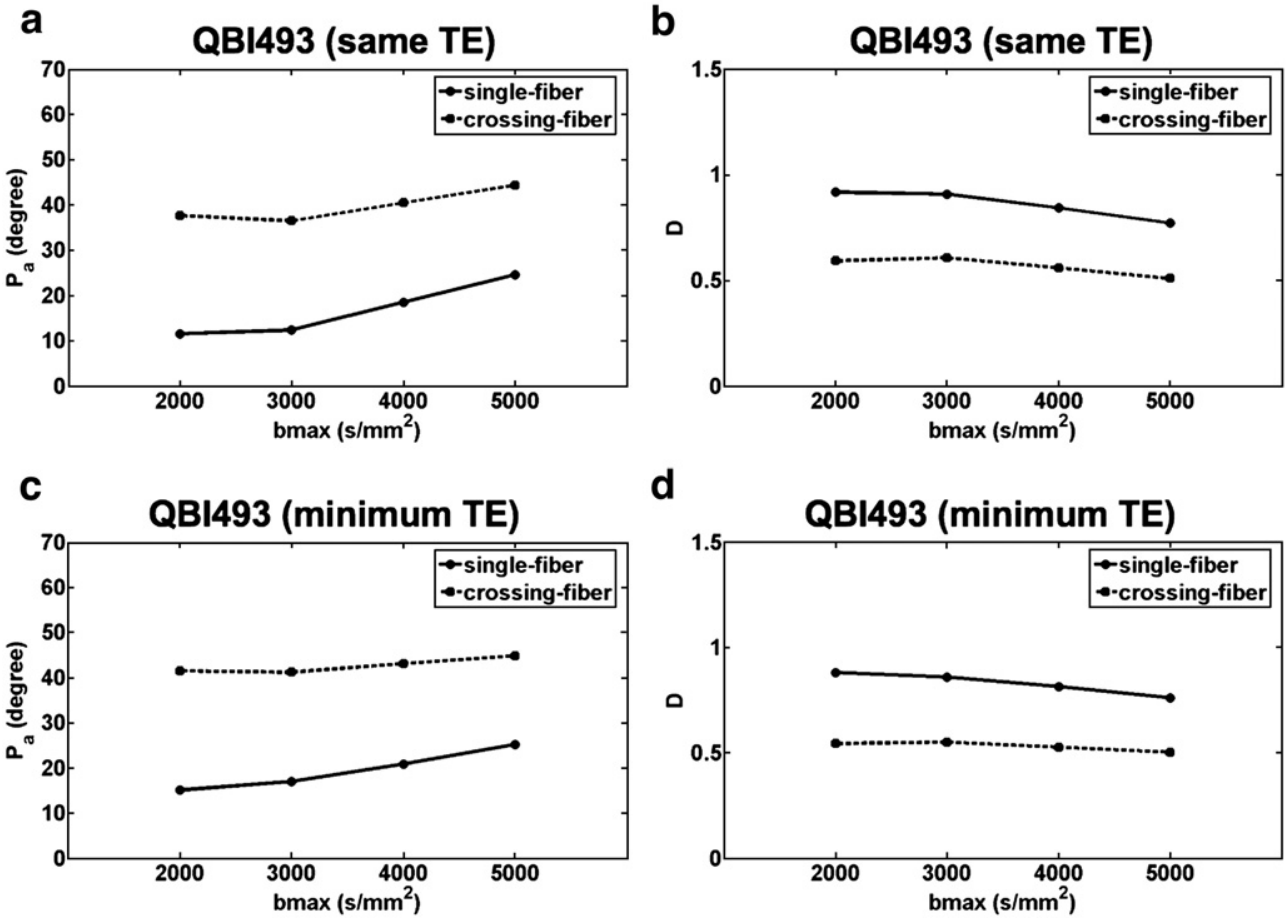

Fig. 7. Angular precision $\left(P_{\mathrm{a}}\right)$ and accuracy $(D)$ against different bmax of QBI493 in the verification study, under same TE (a-b) and minimum TE (c-d). For both cases, a single subject was investigated and the bmax values used in the verification study were 2000, 3000, $4000 \mathrm{and} 5000 \mathrm{~s} / \mathrm{mm}^{2}$.

accuracy than QBI, and the optimum bmax for DSI was higher than that for QBI.

In the simulation, the optimum bmax for DSI to resolve the crossing fibers was generally higher than that for QBI; it was approximately $6000 \mathrm{~s} / \mathrm{mm}^{2}$ for DSI515, 3000 to $4000 \mathrm{~s} / \mathrm{mm}^{2}$ for
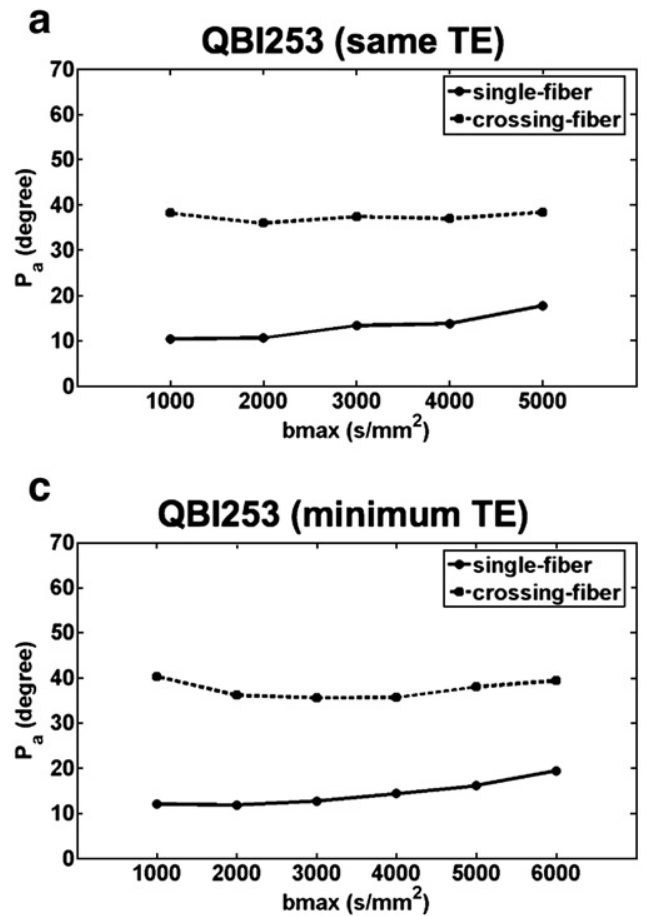

DSI203 and 1000 to $2000 \mathrm{~s} / \mathrm{mm}^{2}$ for QBI493 and QBI253. The simulation results allow us to hone in the range of bmax to be tested in the verification study. Both simulation and verification results showed that they had a consistent tendency; DSI required higher bmax than QBI to achieve optimum condition. The values

b

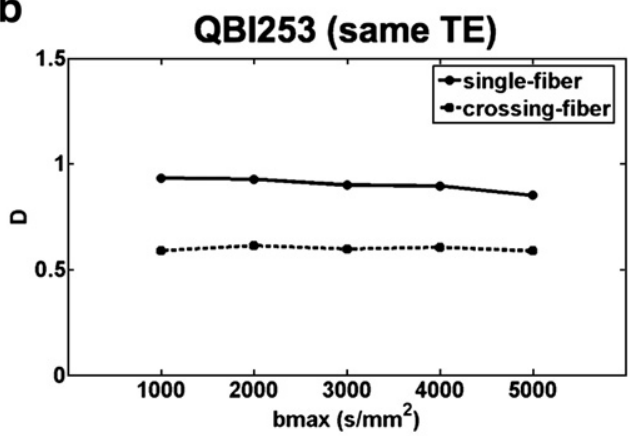

d

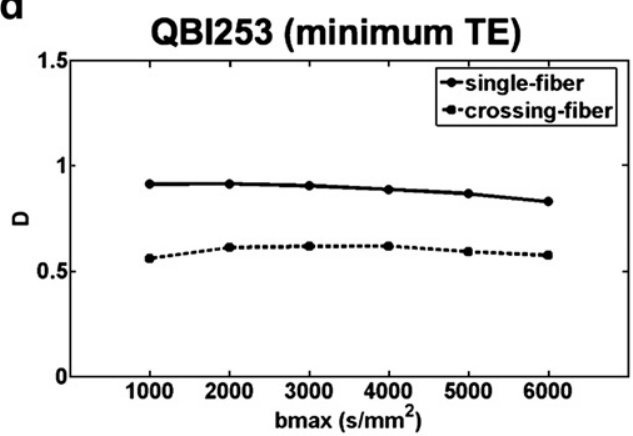

Fig. 8. Angular precision $\left(P_{\mathrm{a}}\right)$ and accuracy $(D)$ against different bmax of QBI253 in the verification study, under same TE (a-b) and minimum TE (c-d). For both cases, a single subject was investigated. The bmax values used in the verification study were 1000 to $5000 \mathrm{~s} / \mathrm{mm}^{2}$ for same TE and 1000 to $6000 \mathrm{~s} / \mathrm{mm}^{2}$ for minimum TE. 


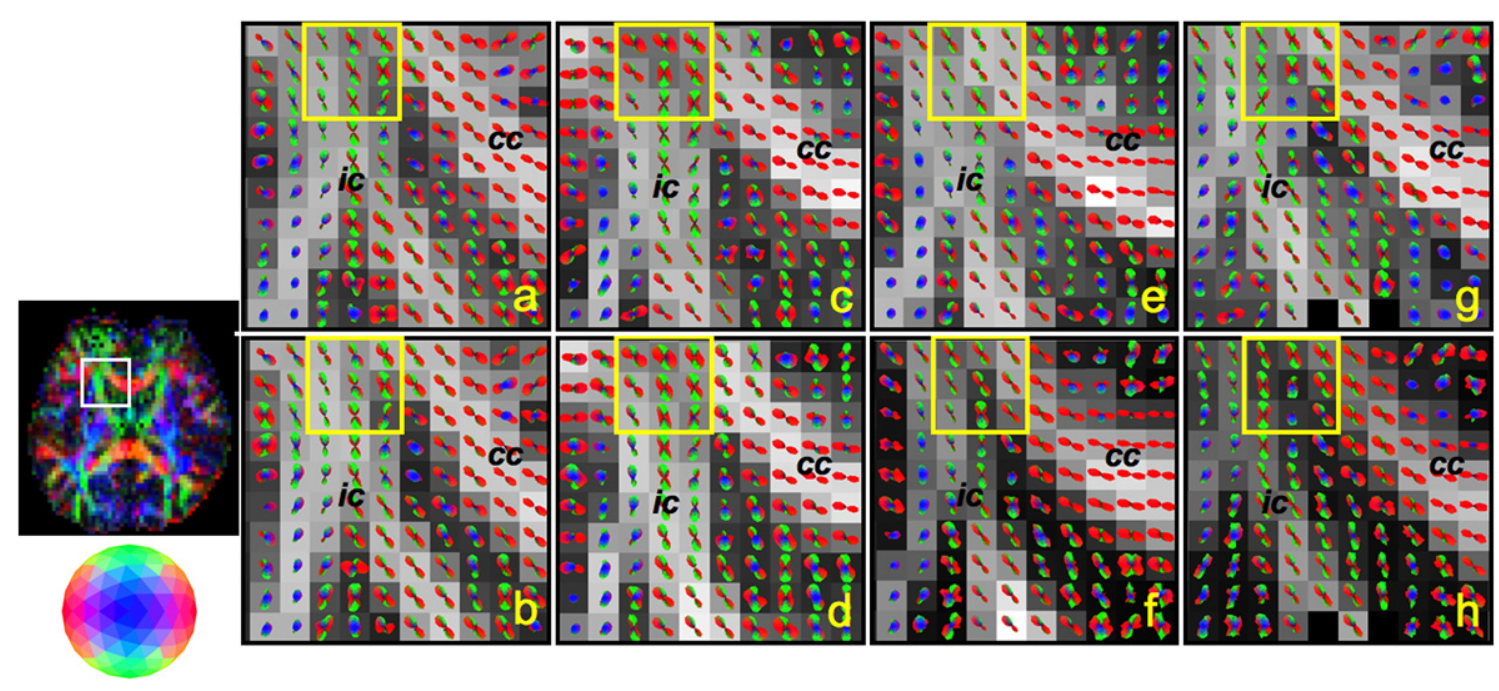

Fig. 9. Intersection between corpus callosum and internal capsule demonstrated with ODF maps reconstructed from the full sampling reference and the corresponding optimum sampling schemes. Panels a and b represent the full sampling reference and the optimum schemes for DSI515; panels c and $\mathrm{d}$ for DSI203; panels e and f for QBI493; panels $g$ and $h$ for QBI253. The full sampling references are in the upper row, the optimum sampling schemes with same TE are in the bottom row. All ODFs were superimposed on the anisotropy maps derived from their corresponding schemes. As shown in the left figure, similar slice position was selected among different sampling schemes. The white open square in the left figure delineates the region equivalent to the zoom-up images from panels a to $\mathrm{h}$. The yellow rectangle in each image encloses pixels showing intersection between corpus callosum (cc) and internal capsule (ic).

of bmax in the verification study, however, were relatively higher than those in the simulation. This may be because SNR was worsened in the simulation when more data at high $b$-values were interpolated, resulting in lower optimum bmax values.

For DSI, higher bmax was proposed to achieve adequate accuracy and angular resolution in human study. As suggested by Wedeen et al. (2005), bmax of 12,000-18,000 s/mm $\mathrm{mm}^{2}$ would be needed. This requirement, however, imposes critical demand on gradient performance and SNR, hence limiting the implementation of DSI in clinical system. In this study, we investigated the bmax from 1000 to $7000 \mathrm{~s} / \mathrm{mm}^{2}$, in which case the diffusion gradients can be operated stably, and the optimum values for different DSI sampling schemes determined. As shown in Fig. 3, DSI515 with lower bmax $\left(1000-2000 \mathrm{~s} / \mathrm{mm}^{2}\right)$ failed to obtain the correct estimation of fiber orientations due to insufficient bmax and source data points for interpolation. Thus, using such low bmax for DSI reconstruction is inappropriate in the simulation study. However, empirically, DSI needs higher bmax than DTI uses (typically $1000-2000 \mathrm{~s} / \mathrm{mm}^{2}$ ) to reconstruct correct PDF profile and resolve fiber orientations. Therefore, the underestimation may not alter our conclusions about the optimum bmax of DSI515 scheme. From our verification results, by averaging the optimum bmax results in both same TE and minimum TE, the optimum bmax was $6500 \mathrm{~s} / \mathrm{mm}^{2}$ for DSI515 and approximately $4000 \mathrm{~s} / \mathrm{mm}^{2}$ for DSI203.

For QBI, the verification study showed that the optimum bmax was $3000 \mathrm{~s} / \mathrm{mm}^{2}$ for QBI493 and $2500 \mathrm{~s} / \mathrm{mm}^{2}$ for QBI253. Our results are consistent with the empirical values used by other groups, the values ranged from 2500 to $4500 \mathrm{~s} / \mathrm{mm}^{2}$ (Khachaturian et al., 2007; Tuch, 2004). In a previous report, Perrin et al. showed that on a clinical scanner QBI could resolve fiber orientation with deviation angles of $15.5^{\circ}$ along the $y$-axis and $19.1^{\circ}$ along the $x$-axis in homogeneous regions and $30^{\circ}$ in crossing regions in a fibercrossing phantom study (Perrin et al., 2005). Our results of in vivo verification study, 8-15 degrees for single fibers and 34-41 degrees for crossing fibers, are reasonable as compared with Perrin's in the phantom study. At the first glance, our angular precision values for single fibers appear slightly lower than Perrin's while our values for crossing fibers slightly higher. This may be due to different metrics used for quantifying the angular deviation. In the single-fiber group, we quantified the angular precision as the mean of deviation angles, whereas Perrin's quantified the angular deviation as the root mean square value of the deviation angles. In the crossing-fiber group, instead of choosing the nearest vectors of the compared data sets as Perrin did, we performed a more stringent comparison by quantifying the deviation angle between the orientations of the first maxima of the two data sets and then the deviation angle between the second maxima. This may lead to a higher deviation angle in our crossing-fiber results.

In the in vivo verification study, there is mis-coregistration error between two data sets due to involuntary motion. This miscoregistration error may further increase the values of angular deviation. If this error is disregarded, the actual values should be within the values obtained from the simulation study and verification study, namely angular precision within $\left\{6^{\circ}, 12^{\circ}\right\}$ for single fibers and within $\left\{27^{\circ}, 36^{\circ}\right\}$ for crossing fibers in QBI253 at bmax of $2500 \mathrm{~s} / \mathrm{mm}^{2}$, and $\left\{4^{\circ}, 10^{\circ}\right\}$ for single fibers and $\left\{23^{\circ}\right.$, $\left.36^{\circ}\right\}$ for crossing fibers in DSI203 at bmax of $4000 \mathrm{~s} / \mathrm{mm}^{2}$.

The verification study showed no significant difference in angular precision and accuracy between DSI515 and DSI203. The major difference between the optimum DSI515 and DSI203 was the values of optimum bmax, which determined the severity of truncation artifact. In this study, we applied the Hanning filter to DSI data before Fourier transform, and it largely reduced the difference in truncation artifact. On the other hand, the sampling rate in the $q$-space was similar for DSI515 and DSI203, resulting in similar FOV in the PDF domain. As compared with DSI203, additional encoding data in DSI515 are acquired in high $b$-values. This would reduce the average SNR of DSI515 and affect the angular resolution. Thus, in the current $3 \mathrm{~T}$ system, we found that DSI515 and DSI203 at their own optimum bmax, $6500 \mathrm{~s} / \mathrm{mm}^{2}$ and 
$4000 \mathrm{~s} / \mathrm{mm}^{2}$, respectively, had comparable angular precision and accuracy. Similarly, in the verification study, especially in the crossing-fiber group, QBI493 and QBI253 at their own optimum bmax values, $3000 \mathrm{~s} / \mathrm{mm}^{2}$ and $2500 \mathrm{~s} / \mathrm{mm}^{2}$, respectively, had comparable angular precision and accuracy. This can be explained by the trade-off between bmax and SNR. Since QBI493 has higher sampling number than QBI253, it allows better angular resolution by trading SNR for a higher value of bmax.

The trade-off between SNR and optimum bmax can be observed in the results of DSI515 in the verification study. Using same TE for different bmax values, it ensures comparable SNR of DSI data; the optimum bmax was found to be $7000 \mathrm{~s} / \mathrm{mm}^{2}$. This finding suggests that with similar SNR and encoding numbers, higher bmax value is preferable for better angular resolution. If minimum TE for different bmax values was used, the scheme with lower bmax could obtain higher SNR owing to shorter TE. The optimum bmax was reduced to $6000 \mathrm{~s} / \mathrm{mm}^{2}$ as a result of the tradeoff between SNR and bmax. Thus, one of the critical factors of the optimum bmax was SNR of DSI data. With sufficient SNR, one should consider a scheme with greater bmax. With insufficient SNR, a scheme with smaller bmax would be preferable to achieve optimum precision and accuracy. In the same vein, if one wants to reduce the gradient number, say from DSI515 to DSI203, but to remain the same bmax, SNR would become too low to provide sufficient angular resolution. As demonstrated in the results, smaller bmax is preferable.

Based on the same principle, the optimum bmax on other MR systems can be deduced from our results. For lower-field systems $(<3 \mathrm{~T})$ or systems with poorer SNR, the optimum bmax is suggested to be smaller than what we found on our current $3 \mathrm{~T}$ system. For higher-field systems or systems with better SNR, such as equipped with head-insert gradient or high-performance RF coil, the optimum bmax is recommended to be greater than our results. Stronger gradient is certainly helpful to diffusion experiments, especially for high angular resolution diffusion imaging schemes (HARDI) which typically require high bmax (Tuch et al., 2002). Compare two hardware settings, one with a weaker gradient strength of $40 \mathrm{mT} / \mathrm{m}$ and the other with a stronger gradient strength of $80 \mathrm{mT} / \mathrm{m}$. Keeping the other factors the same, the diffusion time $\Delta$ can be reduced by a factor of approximately 4 based on the inverse relationship between the square of the gradient strength $g^{2}$ and the diffusion time, i.e., $g^{2} \sim 1 / \Delta$. This would substantially reduce TE, thus gaining SNR. Improvement of SNR can also be achieved by shortening the signal readout using multi-channel acquisition or partial $k$-space readout.

In this study, we recognize that in clinical MRI system the narrow-pulse prerequisite $(\delta<<\Delta)$ for the relationship between diffusion attenuated echo signal and probability density function (PDF) is violated due to the use of finite gradient pulse width. The validity of implementing DSI method in clinical system is, therefore, questioned. Basser pointed out that with comparable diffusion time $(\Delta)$ and gradient duration $(\delta)$, DSI may fail to measure the correct PDF and should provide the same displacement and effective diffusion tensor as DTI technique (Basser, 2002). Wedeen et al. (2005) later investigated the effect of finite gradient pulse width on PDF. Using finite-width diffusion-encoding gradients, the measured displacement was effectively the spin mean position during time interval $[0, \delta]$ relative to the spin mean position during $[\Delta, \Delta+\delta]$. In this case, the displacement may be underestimated and the absolute values of PDF may be altered. However, they claimed that the global shape of the PDF remained and the primary orientation of the diffusion spectrum was substantially unchanged. Lin et al. compared the following two conditions, $\delta<<\Delta$ and $\delta \approx \Delta$, in the rat brains. They showed that the primary orientation of the PDF was not affected by the finite gradient pulse width (Lin et al., 2003b). Besides, when finite gradient pulse duration was used, the accuracy of DSI in defining crossing-fiber orientation was about $6^{\circ}$, close to the noise limit. By comparing the experiments with short and long gradient durations, they found that the bias was approximately $10^{\circ}$. Based on the above argument and evidence, we believe that DSI can be used to map primary orientations of PDF even with finite gradient pulse duration.

In this paper, we showed that it is possible to reduce the gradient numbers of DSI or QBI to keep the scan time and gradient stability within acceptable limits. With the optimum values of bmax, our results showed that both DSI and QBI schemes of less than 300 encoding points could achieve angular resolution comparable to those using approximately 500 points. The scan time of DSI203 or QBI253 for whole brain scanning can be reduced to around $30 \mathrm{~min}$. Further reduction of the encoding number can be accomplished by hemi-sphere or hemi-shell acquisition over the $q$-space (Lin et al., 2003a) or by a non-Cartesian sampling scheme (BCC) at body center cubic (Chiang et al., 2006). Using BCC sampling scheme, a sparser sampling density ( $\sim 70 \%$ of the Cartesian sampling scheme) can be used to obtain PDF with less aliasing artifact encountered in Fourier transform. This benefit may turn into improvement of angular resolution in HARDI experiment. The advancement of fast sequences such as simultaneous echo refocusing (SER) EPI or modern parallel imaging techniques are other potential techniques that can be used simultaneously with the above reduced sampling schemes (Jaermann et al., 2004; Reese et al., 2006; Skare et al., 2007).

Under current hardware setting ( $3 \mathrm{~T}$ without parallel imaging capability), we recommend to use DSI203 instead of typical DSI515 for the whole brain acquisition. In our system, the shortest TR for the whole brain acquisition is approximately 8 to $9 \mathrm{~s}$, thus the total scan time for DSI203 acquisition is approximately 27-30 min. In our results, DSI203 can achieve comparable angular precision and accuracy as DSI515. Therefore, DSI203 is potentially applicable for clinical use and acceptable accuracy can be achieved under the optimum bmax we found on our current 3 T MRI system, i.e., $4000 \mathrm{~s} / \mathrm{mm}^{2}$. If DSI sequence is not available, we recommend QBI253 or fewer encoding points, approximately 200 points, as an alternative. Although we found that under comparable scan time DSI has incremental gain in angular precision and accuracy compared to QBI, the optimum bmax for QBI (about $2500 \mathrm{~s} / \mathrm{cm}^{2}$ ) is lower than that for DSI (about $4000 \mathrm{~s} / \mathrm{cm}^{2}$ ). This means that the gradient performance of QBI is less demanding than DSI.

In this paper, we investigated the optimum bmax for the clinically feasible DSI and QBI sampling schemes on a 3 T clinical scanner. With optimum setting under performance achievable currently, both DSI and QBI schemes could attain comparable angular precision of approximately $8^{\circ}$ for single fibers and $30^{\circ}$ for crossing fibers. The tractography produced from such schemes can constantly show crossing of major tracts, for instance, in the centrum semiovale where corona radiata, superior longitudinal fasciculus and corpus callosum intersect, in the pons where middle cerebellar peduncle and corticospinal tract intersect, and in the boundary between cingulum and corpus callosum. These optimum sampling schemes, however, are limited in resolving crossing at narrow angles or crossing of two tracts with disproportional volumes. Recent advance in high performance gradient coils, multi-channel parallel RF acquisition and single shot echo volume imaging techniques could improve the angular resolution 
of high angular $q$-space diffusion imaging and maintain sufficient SNR. This could potentially resolve more crossing and finer tracts.

The present study investigated the optimum bmax for different sampling numbers of DSI and QBI. On our current 3 T system, the optimum bmax was $6500 \mathrm{~s} / \mathrm{mm}^{2}$ for DSI515 and $4000 \mathrm{~s} / \mathrm{mm}^{2}$ for DSI203; it was $3000 \mathrm{~s} / \mathrm{mm}^{2}$ for QBI493 and $2500 \mathrm{~s} / \mathrm{mm}^{2}$ for QBI253. Under comparable scan time, our results showed that DSI had an incremental advantage in angular resolution over QBI. DSI, however, required a higher demand on gradient performance. In conclusion, our systematic study of optimum bmax in different sampling schemes and the consideration derived wherein could be helpful to determine optimum sampling schemes in other MRI systems.

\section{Acknowledgments}

The work was supported in part by the grant NSC95-2752-M-002018-PAE. We are grateful to Mr. Chandan Mishra for his assistance in data analysis and Dr. Kai-Hsiang Chuang for his helpful comments.

\section{References}

Basser, P.J., 2002. Relationships between diffusion tensor and $q$-space MRI. Magn. Reson. Med. 47, 392-397.

Basser, P.J., Mattiello, J., LeBihan, D., 1994. MR diffusion tensor spectroscopy and imaging. Biophys. J. 66, 259-267.

Callaghan, P.T., 1991. Principles of Nuclear Magnetic Resonance Microscopy. Clarendon Press, Oxford.

Chiang, W.Y., Wedeen, V.J., Kuo, L.W., Peng, M.H., Tseng, W.Y., 2006. Diffusion spectrum imaging using Body-Center-Cubic sampling scheme. Proceedings of the 14th Meeting of the International Society for Magnetic Resonance in Medicine. ISMRM, Berkeley, Seattle, USA, p. 1041.

Ciccarelli, O., Behrens, T.E., Altmann, D.R., Orrell, R.W., Howard, R.S., Johansen-Berg, H., Miller, D.H., Matthews, P.M., Thompson, A.J., 2006. Probabilistic diffusion tractography: a potential tool to assess the rate of disease progression in amyotrophic lateral sclerosis. Brain 129, 1859-1871.

Frank, L.R., 2001. Anisotropy in high angular resolution diffusion-weighted MRI. Magn. Reson. Med. 45, 935-939.

Frank, L.R., 2002. Characterization of anisotropy in high angular resolution diffusion-weighted MRI. Magn. Reson. Med. 47, 1083-1099.

Ge, Y., Law, M., Grossman, R.I., 2005. Applications of diffusion tensor MR imaging in multiple sclerosis. Ann. N.Y. Acad. Sci. 1064, 202-219.

Gilbert, R.J., Magnusson, L.H., Napadow, V.J., Benner, T., Wang, R., Wedeen, V.J., 2006a. Mapping complex myoarchitecture in the bovine tongue with diffusion-spectrum magnetic resonance imaging. Biophys. J. 91, 1014-1022.

Gilbert, R.J., Wedeen, V.J., Magnusson, L.H., Benner, T., Wang, R., Dai, G., Napadow, V.J., Roche, K.K., 2006b. Three-dimensional myoarchitecture of the bovine tongue demonstrated by diffusion spectrum magnetic resonance imaging with tractography. Anat. Rec. A, Discov. Mol. Cell. Evol. Biol. 288, 1173-1182.

Hagmann, P., Jonasson, L., Maeder, P., Thiran, J.P., Wedeen, V.J., Meuli, R., 2006. Understanding diffusion MR imaging techniques: from scalar diffusion-weighted imaging to diffusion tensor imaging and beyond. Radiographics 26 (Suppl 1), S205-S223.

Hagmann, P., Kurant, M., Gigandet, X., Thiran, P., Wedeen, V.J., Meuli, R., Thiran, J.P., 2007. Mapping human whole-brain structural networks with diffusion MRI. PLoS ONE 2, e597.

Jaermann, T., Crelier, G., Pruessmann, K.P., Golay, X., Netsch, T., van Muiswinkel, A.M., Mori, S., van Zijl, P.C., Valavanis, A., Kollias, S., Boesiger, P., 2004. SENSE-DTI at 3 T. Magn. Reson. Med. 51, 230-236.

Jiang, H., Golay, X., van Zijl, P.C., Mori, S., 2002. Origin and minimization of residual motion-related artifacts in navigator-corrected segmented diffusion-weighted EPI of the human brain. Magn. Reson. Med. 47, 818-822.

Jones, D.K., Catani, M., Pierpaoli, C., Reeves, S.J., Shergill, S.S., O’Sullivan, M., Golesworthy, P., McGuire, P., Horsfield, M.A., Simmons, A.,
Williams, S.C., Howard, R.J., 2006. Age effects on diffusion tensor magnetic resonance imaging tractography measures of frontal cortex connections in schizophrenia. Hum. Brain Mapp. 27, 230-238.

Khachaturian, M.H., Wisco, J.J., Tuch, D.S., 2007. Boosting the sampling efficiency of $q$-Ball imaging using multiple wavevector fusion. Magn. Reson. Med. 57, 289-296.

Kubicki, M., McCarley, R., Westin, C.F., Park, H.J., Maier, S., Kikinis, R., Jolesz, F.A., Shenton, M.E., 2007. A review of diffusion tensor imaging studies in schizophrenia. J. Psychiatr. Res. 41, 15-30.

Kuo, L.W., Song, S.K., Wedeen, V.J., Lin, C.P., Chen, J.H., Tseng, W.Y., 2003. Mean diffusivity and anisotropy index mapping of diffusion spectrum imaging in a stroke model. Proceedings of the 11th Meeting of the International Society for Magnetic Resonance in Medicine. ISMRM, Berkeley, Toronto, Canada, p. 592.

Le Bihan, D., 2003. Looking into the functional architecture of the brain with diffusion MRI. Nat. Rev. Neurosci. 4, 469-480.

Le Bihan, D., Poupon, C., Amadon, A., Lethimonnier, F., 2006. Artifacts and pitfalls in diffusion MRI. J. Magn. Reson. Imaging 24, 478-488.

Lin, C.P., Tseng, W.Y., Weng, J.C., Wedeen, V.J., Chen, J.H., 2003a. Reduced encoding of diffusion spectrum imaging with cross-term correction. Proceedings of the 1st IEEE EMBS Conference on Neural Engineering. IEEE Publishing Co., Piscataway, Capri Island, Italy, pp. 561-563.

Lin, C.P., Wedeen, V.J., Chen, J.H., Yao, C., Tseng, W.Y., 2003b. Validation of diffusion spectrum magnetic resonance imaging with manganese-enhanced rat optic tracts and ex vivo phantoms. Neuroimage 19, 482-495.

Mardia, K.V., Jupp, P.E., 2000. Directional Statistics, 2. J. Wiley, Chichester.

Meca, C., Chabert, S., Le Bihan, D., 2004. Diffusion MRI at large b values: what's the limit. Proceedings of the 12th Meeting of the International Society for Magnetic Resonance in Medicine. ISMRM, Berkeley, Kyoto, Japan, p. 1196.

Mori, S., van Zijl, P.C., 2002. Fiber tracking: principles and strategies-a technical review. NMR. Biomed. 15, 468-480.

Perrin, M., Poupon, C., Rieul, B., Leroux, P., Constantinesco, A., Mangin, J.F., Lebihan, D., 2005. Validation of $q$-ball imaging with a diffusion fibrecrossing phantom on a clinical scanner. Philos. Trans. R. Soc. Lond., B Biol. Sci. 360, 881-891.

Pierpaoli, C., Jezzard, P., Basser, P.J., Barnett, A., Di Chiro, G., 1996. Diffusion tensor MR imaging of the human brain. Radiology 201, 637-648.

Reese, T.G., Heid, O., Weisskoff, R.M., Wedeen, V.J., 2003. Reduction of eddy-current-induced distortion in diffusion MRI using a twicerefocused spin echo. Magn. Reson. Med. 49, 177-182.

Reese, T.G., Benner, T., Wang, R., Feinberg, D.A., Wedeen, V.J., 2006. Halving imaging time of whole brain diffusion spectrum imaging (DSI) by using simultaneous echo refocusing (SER) EPI. Proceedings of the 14th Meeting of the International Society for Magnetic Resonance in Medicine. ISMRM, Berkeley, Seattle, USA, p. 1044.

Schmahmann, J.D., Pandya, D.N., Wang, R., Dai, G., D'Arceuil, H.E., de Crespigny, A.J., Wedeen, V.J., 2007. Association fibre pathways of the brain: parallel observations from diffusion spectrum imaging and autoradiography. Brain 130, 630-653.

Skare, S., Newbould, R.D., Clayton, D.B., Albers, G.W., Nagle, S., Bammer, R., 2007. Clinical multishot DW-EPI through parallel imaging with considerations of susceptibility, motion, and noise. Magn. Reson. Med. 57, 881-890.

Tuch, D.S., 2004. $Q$-ball imaging. Magn. Reson. Med. 52, 1358-1372.

Tuch, D.S., Reese, T.G., Wiegell, M.R., Makris, N., Belliveau, J.W., Wedeen, V.J., 2002. High angular resolution diffusion imaging reveals intravoxel white matter fiber heterogeneity. Magn. Reson. Med. 48, 577-582.

Tuch, D.S., Reese, T.G., Wiegell, M.R., Wedeen, V.J., 2003. Diffusion MRI of complex neural architecture. Neuron 40, 885-895.

Tuch, D.S., Wisco, J.J., Khachaturian, M.H., Ekstrom, L.B., Kotter, R., Vanduffel, W., 2005. $Q$-ball imaging of macaque white matter architecture. Philos. Trans. R. Soc. Lond., B. Biol. Sci. 360, 869-879.

Wedeen, V.J., Hagmann, P., Tseng, W.Y., Reese, T.G., Weisskoff, R.M., 2005. Mapping complex tissue architecture with diffusion spectrum magnetic resonance imaging. Magn. Reson. Med. 54, 1377-1386.

Wiegell, M.R., Larsson, H.B., Wedeen, V.J., 2000. Fiber crossing in human brain depicted with diffusion tensor MR imaging. Radiology 217, 897-903. 